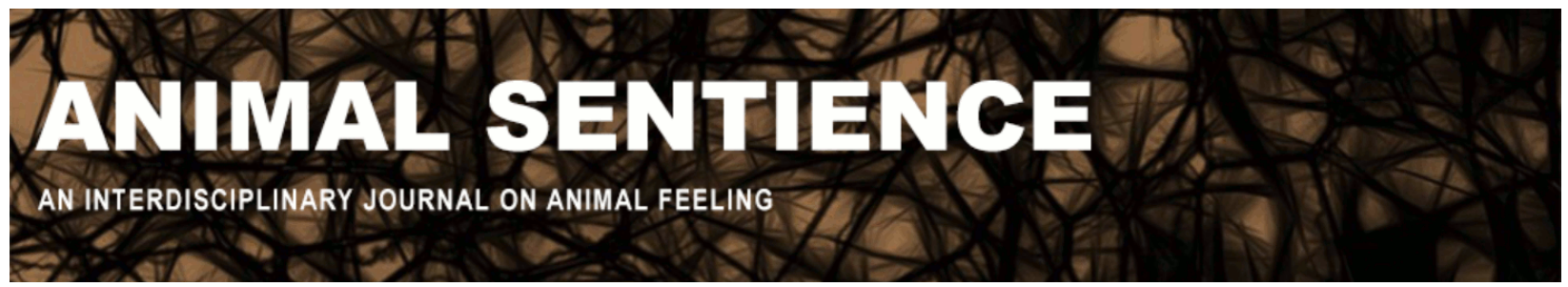

Martin, Loren and Gerlai, Robert (2018) Sentience: All or none or matter of degree?. Animal Sentience 21(9)

DOI: $10.51291 / 2377-7478.1343$

Date of submission: 2018-06-08

Date of acceptance: 2018-06-15 (c) 


\title{
Sentience: All or none or matter of degree?
}

Commentary on Sneddon et al. on Sentience Denial

\author{
Loren Martin \& Robert Gerlai \\ Department of Psychology \\ University of Toronto Mississauga
}

\begin{abstract}
The question of whether fish feel pain is muddied by anthropomorphic thinking. Comparing biological phenomena in two species should be informed by the criteria for good animal models: face validity, construct validity and predictive validity. Viewed through this lens, we argue that fish do feel pain and may possess some level of sentience. Evolutionary relatedness, hence similarities and differences between species (fish and humans in this case), are not about black vs. white but about shades of grey.
\end{abstract}

Loren Martin is assistant professor of behavioral neuroscience and Canada Research Chair in Translational Pain Research. His lab explores how chronic pain changes brain circuitry so that the circuits that encode pain relief can be identified. He has received early career recognition from both the Canadian Pain and American Pain Societies. Website

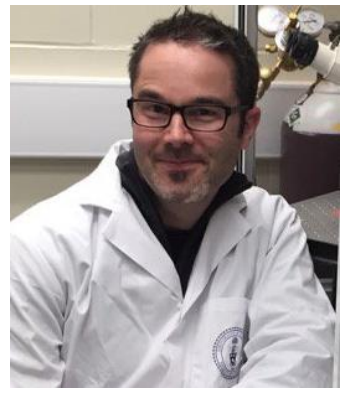

Robert Gerlai is professor of behavioral neuroscience and studies neurobiological and genetic mechanisms of behavior in fish and rodents. He is Fellow and past president of the IBNS. He received the Distinguished Scientist Award from IBANGS in 2013 and the University of Toronto Mississauga Excellence in Research Award in 2015. Website

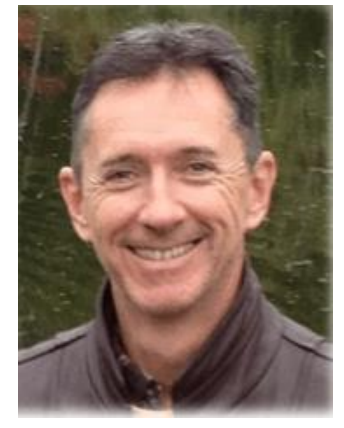

Introduction. Sneddon et al. (2018) make several excellent points about why feeling pain should not be viewed as a fundamentally different phenomenon in fish compared to higher order vertebrates such as mammals, including our own species. They touch on many complex questions about what it is to feel pain or be aware of pain or be sentient. They also discuss how to measure and interpret pain-induced behavioral responses in fish. The problems are similar to those raised in this journal by the prior target articles on fish sentience by Woodruff in 2017 and Key in 2016. The current target article is placed in the context of animal welfare and whether fish deserve the same attention as mammals in this domain.

Can animals feel pain? A question with many practical consequences. The importance of painful or aversive conditions and stimuli extends beyond the question of animal welfare. They can have 
significant effects on how animals respond to experimental manipulations. If these effects are ignored, the results and interpretations may be biased. For example, zebrafish have recently been found to exhibit a paradoxical reduction of whole-body cortisol levels in response to isolation (Shams et al., 2017); it has also been shown that their cortisol levels increase under painful or stressful stimulation (Shams et al., 2017; 2018). So, is isolation a "good thing" for the zebrafish, a species known for its strong shoaling tendency (Miller \& Gerlai, 2011)? Further examination suggests that the answer is no (Shams et al., 2017). The isolated fish must have had reduced cortisol levels compared to control non-isolated fish, who were stressed under the standard housing conditions (Shams et al., 2017): the high density crowding in the small fish tanks and rack systems preferred commercially (and in the zebrafish community).

A similar realization was mentioned at a recent zebrafish meeting organized by $\mathrm{NIH}$ for discussing the optimal environmental conditions for maintaining and testing zebrafish. At that meeting, Stephen Ekker (editor of the journal Zebrafish) reported unpublished results (of Karl Clark) from his institute showing that the standard 96-well plate in which larval zebrafish are usually tested induces significant stress responses, as compared to when the fish are placed on the 24-well plate, in which the wells provide a larger volume of water per fish and thus more space to move around (personal communication with permission from Ekker \& Clark). Since stress, fear and anxiety can reduce pain behavior through stress-induced analgesia, environmental factors such as housing or testing conditions can have a great impact on the interpretation of experimental findings in both rodents and fish (see Butler \& Finn, 2009, for a review).

What does it mean to be aware of having pain? Are fish aware of their stress? do they experience pain? do they experience fear or panic? and do they experience it the way we humans do? First, let us consider the human infant. Babies are incapable of communicating verbally how much pain they are in or where it hurts. Should this lead us to believe that they do not experience pain? We must interpret their cries (pitch, loudness, timbre) and global behavior as proxies for pain. Shockingly, in the 1980s, a common belief among medical doctors, especially surgeons, was that human infants did not have the neural circuity sufficient to process pain signals (see McGraw, 1941, for the original paper that formed the basis for this dubious thinking). As a result, infants undergoing surgery were not administered anesthesia or analgesic medications - only a paralytic agent so that the surgeon could perform the necessary procedure (McGrath, 2011). Of course, this is outdated thinking, and now there is a whole sub-field of pain research devoted to minimizing pain and distress in infants (Hall \& Anand, 2014). We have realized our thinking was wrong and we have changed it.

Our thinking has been slower to change with respect to animal pain and distress behavior. In principle, the criteria for properly examining whether fish or other animals exhibit stress, pain or fear are not that different from how one would evaluate the responses of infants or non-verbal humans. In babies, facial expressions have been validated as a reliable pain assessment tool, and this same approach has been successfully used in mice, rats, horses, dogs, cats and other species (Dalla Costa et al., 2016; Langford et al., 2010; Reijgwart et al., 2017; Sotocinal et al., 2011). Unfortunately for fish, they do not have the capacity for changing their facial expression, at least not in a way that would be evident to the human observer. This may have led some to conclude that lower order animals do not experience pain. Although fish may not have clearly apparent 
facial expressions (but see anabantids, e.g., the Siamese fighting fish's aggressive gill erection display), they do exhibit a rich repertoire of motor/movement patterns - "gestures" that one may be able to interpret as reflecting their emotional state or other aspects of their brain function (Kalueff et al., 2013). Similarly, as pointed out in the target article, fish respond in a predictable way to algogens (i.e., pain-producing substances) when exposed via the holding water (LopezLuna, Al-Jubouri, Al-Nuaimy, \& Sneddon, 2017) or injected (Taylor et al., 2017). The typical response is a reduction in locomotor activity, easily quantified as diminished distance traveled in zebrafish larvae/adults. This behavior appears to be an evolutionarily conserved response in numerous species including mammals (Pitzer et al., 2016). Developing an accurate measure for "fish-pain" may require more work, but decreased swimming activity already appears to be a reasonable outcome measure. The observation that administration of the common analgesic morphine reverses algogen effects on distance travelled in zebrafish (Taylor et al., 2017) supports this.

Pain assessment tools, whether in fish, birds, rodents or dogs, are based on what some call "reflexive" responses: We apply a stimulus (mechanical, thermal or chemical) and measure the response, usually force applied, thermal threshold or time to respond (Mogil, 2009). While rodents are the preeminent choice of subject for the majority of biomedical research, they are prey species (especially the mouse) and have a tendency to "mask" their pain when predators are in the same room, including the human experimenter (Sorge et al., 2014). Nerve-injured animals often appear to walk just fine, and postoperative mice recover exceedingly quickly (both in terms of reflexive pain assessment and spontaneous measures). Mice actually recover in ways that would seem "super-human," yet we are less likely to label rodents as incapable of pain processing. For animals, behavior must be interpreted in the context of the species and through a holistic evolutionary lens. Fish do not communicate pain in a way that is simple and understandable to humans. We measure and interpret their behavioral responses, but should fish be categorized as reflexive automata? Most people would agree that rodents experience some degree of pain; we label their distress as pain, because these species look more expressive to the anthropomorphic observer. Unlike fish, rodents vocalize and have better-defined behavioral pain responses that are similar to our own.

Face validity, construct validity and predictive validity. Fish are relatively understudied in the neurosciences. Building a behavioral repertoire in fish has only started recently (Kalueff et al., 2013). How can we decide about similarities and differences across species like the zebrafish and humans? How can we tell whether fish respond to and feel pain? The answer is not fundamentally different from what most scientists struggle with when they try to mimic or model human brain functions or dysfunctions using animals. What makes a good animal model? The criteria are threefold: face validity, construct validity and predictive validity.

Face validity refers to the requirement that the animal should appear to respond to the experimental treatment similarly to the way a human would. This is, in effect, a kind of Turing test for biomedical research. Although fish are separated from humans by about 400 million years of biological evolution, and although they do differ from us at practically every level of biological organization, when painful or otherwise aversive stimulation is applied, just like humans, they respond in a way that is often opposite to what a pleasurable, rewarding or otherwise positive stimulus/treatment would induce. There are numerous examples of such responses at the 
behavioral and neural levels, down to the level of gene expression. Many are reviewed in Sneddon et al.'s target article, leaving no doubt that the appearance of the pain response in fish is similar to what one would expect in humans and other non-human mammalian species.

As one goes deeper in the levels of biological organization, from behavior down towards genes, one is entering the domain of the second criterion: construct validity: Does pain induce similar brain responses in fish and humans? Are the changes in the brains of fish and humans associated with similar molecular interactions and biochemical pathways? The answer is again nicely treated in Sneddon et al.'s target article: Although many differences and controversies exist in the literature, there is sufficient evidence of similarities in biological mechanisms underlying pain perception, pain processing and pain responses in fish and humans.

This leads to the third criterion, predictive validity, which is defined slightly differently depending on research context. In drug development by the pharmaceutical research industry, predictive validity concerns whether one can use the animal model to identify small molecules that would work the same way in humans: Could the animal model predict drug efficacy? This hinges directly on mechanistic similarity. If the molecular components of the biological (behavioral) phenomenon - such as pain perception, processing and responding - are at least partly shared between fish and humans, the same drugs that bind to the fish molecular target and alter its function should also act on corresponding human molecular targets. The reciprocal effect has already been shown: several human drugs work quite well (i.e., induce similar functional changes) in zebrafish (Stewart et al., 2014; Kalueff et al., 2014). This is not just an academic discussion: pharmaceutical companies are investing millions of dollars in zebrafish and using this small, prolific, and hence cheap organism for their drug-screening efforts (Fleming \& Alderton, 2013).

Similarities and differences. How can one judge similarities and differences across evolutionarily distant species like fish and mammals? The target article does a good job in emphasizing the similarities. In most of our own zebrafish papers, we emphasize such similarities too. However, this may represent wishful thinking rather than objectivity. We do not dispute the importance of similarities. Most, if not all, existing species are related to each other; thus, some level of similarity, i.e., evolutionary conservation, is inevitable. How we can evaluate the degree of relatedness, evolutionary conservation, or similarity? This is relatively simple at the level of the genome. One can calculate the percentage of homology between the nucleotide sequences of fish and corresponding human genes, the level of synteny (order of genes on the chromosome) between these species, and the amino-acid sequence homology at the level of proteins. One can even study homology focusing on the most important binding pockets of proteins. At this level of biological organization, the similarity between zebrafish and human amounts to about $70 \%$ nucleotide sequence homology, and a similar percentage of synteny and proportion of genes having homologs in the two species. The amino acid sequence homology of binding pockets of proteins can reach an even higher value, approaching or exceeding $90 \%$ in some cases. From a molecular biological viewpoint, fish and humans are clearly not that different. But what does this mean from the perspective of complex behaviors, including fear, stress, and pain-related phenomena? Quantifying similarity at this level is quite difficult at best, and quite subjective at worst. 
Black and white or shades of grey? Framing our questions in the form of "complex behavior vs. reflexive automatism," "sentience vs. insentience," and "feeling pain as we do vs. not" will lead to misleading answers. We are not even sure that mice and rats process pain/nociceptive signals the same way we do (Mogil, 2009). Evolutionary relatedness or homologous responses do not mean sameness. Categorization and dichotomization are inherent to the way humans - scientists and nonscientists - think. But given what we know about evolution and the considerable information that biologists have accumulated across a large number of species, biological similarity, and hence also species differences, are matters of degree. As we argued in a previous commentary about sentience and consciousness (Gerlai, 2017), pain-related phenomena are likely to follow a similar pattern: We have no doubt that fish, like all animals, feel pain. The question is: How similar is this feeling to what humans feel?

\section{References}

Butler, R. K., \& Finn, D. P. (2009). Stress-induced analgesia. Progress in Neurobiology, 88(3), 184202.

Dalla Costa, E., Stucke, D., Dai, F., Minero, M., Leach, M. C., \& Lebelt, D. (2016). Using the Horse Grimace Scale (HGS) to assess pain associated with acute laminitis in horses (Equus caballus). Animals (Basel), 6(8).

Fleming, A., \& Alderton, W. K. (2013). Zebrafish in pharmaceutical industry research: Finding the best fit. Drug Discovery Today: Disease Models, 10(1), e43-e50.

Gerlai, R. (2017). Learning, memory, cognition, and the question of sentience in fish. Animal Sentience 13(8).

Hall, R. W., \& Anand, K. J. (2014). Pain management in newborns. Clinics in Perinatology, 41(4), 895-924.

Kalueff, A. V., Gebhardt, M., Stewart, A. M., Cachat, J. M., Brimmer, M., Chawla, J. S., Craddock, C., Kyzar, E. J., Roth, A., Landsman, S., Gaikwad, S., Robinson, K., Baatrup, E., Tierney, K., Shamchuk, A., Norton, W., Miller, N., Nicolson, T., Braubach, O., Gilman, C. P., Pittman, J., Rosemberg, D. B., Gerlai, R., Echevarria, D., Lamb, E., Neuhauss, S. C. F., Weng, W., BallyCuif, L., Schneider, H., \& the Zebrafish Neuroscience Research Consortium. (2013). Towards a comprehensive catalog of zebrafish behavior 1.0, and beyond. Zebrafish, 10, 70-86.

Kalueff, A. V., Stewart, A. M., \& Gerlai, R. (2014). Zebrafish as an emerging model for studying complex brain disorders. Trends in Pharmacological Sciences, 35, 63-75.

Key, B. (2016). Why fish do not feel pain. Animal Sentience 3(1).

Langford, D. J., Bailey, A. L., Chanda, M. L., Clarke, S. E., Drummond, T. E., Echols, S., Glick, S., Ingrao, J., Klassen-Ross, T., LaCroix-Fralish, M. L., Matsumiya, L., Sorge, R. E., Sotocinal, S. G., Tabaka, J. M., Wong, D., van den Maagdenberg, A. M. J. M., Ferrari, M. D., Craig, K. D., \& Mogil, J. S. (2010). Coding of facial expressions of pain in the laboratory mouse. Nature Methods, 7(6), 447-449.

Lopez-Luna, J., Al-Jubouri, Q., Al-Nuaimy, W., \& Sneddon, L. U. (2017). Reduction in activity by noxious chemical stimulation is ameliorated by immersion in analgesic drugs in zebrafish. Journal of Experimental Biology, 220(8), 1451-1458. 
McGrath, P. J. (2011). Science is not enough: The modern history of pediatric pain. Pain, 152(11), 2457-2459.

McGraw, M. (1941). Neural maturation as exemplified in the changing reactions of the infant to pin prick. Child Development, 12(1), 31-42.

Miller, N., \& Gerlai, R. (2011). Shoaling in zebrafish: What we don't know. Reviews in the Neurosciences, 22, 17-25.

Mogil, J. S. (2009). Animal models of pain: Progress and challenges. Nature Reviews Neuroscience, 10(4), 283-294.

Pitzer, C., Kuner, R., \& Tappe-Theodor, A. (2016). Voluntary and evoked behavioral correlates in inflammatory pain conditions under different social housing conditions. Pain Reports, 1(1), e564.

Reijgwart, M. L., Schoemaker, N. J., Pascuzzo, R., Leach, M. C., Stodel, M., de Nies, L., Hendriksen, C. F. M., van der Meer, Vinke, C. M., \& van Zeeland, Y. R. A. (2017). The composition and initial evaluation of a grimace scale in ferrets after surgical implantation of a telemetry probe. PLoS One, 12(11), e0187986.

Shams, S., Rihel J., Ortiz, J. G., \& Gerlai, R. (2018). The zebrafish as a promising tool for modeling human brain disorders: A review based upon an IBNS Symposium. Neuroscience \& Biobehavioral Reviews, 85, 176-190.

Shams, S., Seguin, D., Facciol, A., Chatterjee, D., \& Gerlai, R. (2017). Effect of social isolation on anxiety-related behaviors, cortisol, and monoamines in adult zebrafish. Behavioral Neuroscience, 131(6), 492-504.

Sneddon, L. U., Lopez-Luna, J., Wolfenden, D. C. C., Leach, M. C., Valentim, A. M., Steenbergen, P. J., Bardine, N., Currie, A. D., Broom, D. M., \& Brown, C. (2018). Fish sentience denial: Muddying the waters. Animal Sentience 21(1).

Sorge, R. E., Martin, L. J., Isbester, K. A., Sotocinal, S. G., Rosen, S., Tuttle, A. H., Wieskopf, J. S., Acland, E. L., Dokova, A., Kadoura, B., Leger, P., Mapplebeck, J. C., McPhail, M., Delaney, A., Wigerblad, G., Schumann, A. P., Quinn, T., Frasnelli, J., Svensson, C. I., Sternberg, W. F., \& Mogil, J. S. (2014). Olfactory exposure to males, including men, causes stress and related analgesia in rodents. Nature Methods, 11(6), 629-632.

Sotocinal, S. G., Sorge, R. E., Zaloum, A., Tuttle, A. H., Martin, L. J., Wieskopf, J. S., Mapplebeck, J. C. S., Wei, P., Zhan, S., Zhang, S., McDougall, J. J., King, O. D., \& Mogil, J. S. (2011). The Rat Grimace Scale: A partially automated method for quantifying pain in the laboratory rat via facial expressions. Molecular Pain, 7, 55.

Stewart, A. M., Braubach, O., Spitsbergen, J., Gerlai, R., \& Kalueff, A. (2014). Zebrafish models for translational neuroscience research: From tank to bedside. Trends in Neurosciences, 37, 264-278.

Taylor, J. C., Dewberry, L. S., Totsch, S. K., Yessick, L. R., DeBerry, J. J., Watts, S. A., \& Sorge, R. E. (2017). A novel zebrafish-based model of nociception. Physiology \& Behavior, 174, 83-88.

Woodruff, M. L. (2017). Consciousness in teleosts: There is something it feels like to be a fish. Animal Sentience 13(1). 


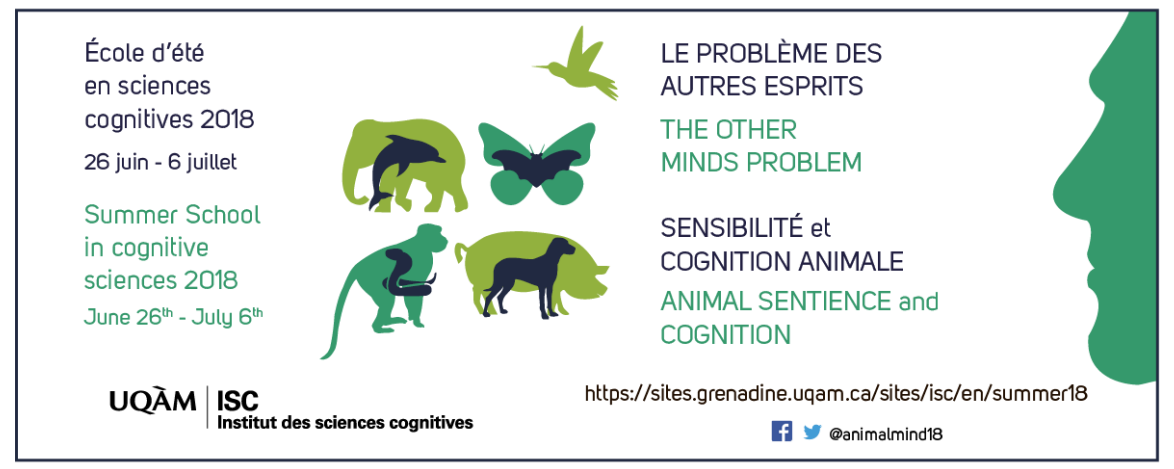

ISC 2018 Summer School in Cognitive Sciences June 26 - July 6, 2018 Montreal (Canada)

\section{The Other Minds Problem: Animal Sentience and Cognition}

Overview. Since Descartes, philosophers know there is no way to know for sure what — or whether - others feel (not even if they tell you). Science, however, is not about certainty but about probability and evidence. The 7.5 billion individual members of the human species can tell us what they are feeling. But there are 9 million other species on the planet $(20$ quintillion individuals), from elephants to jellyfish, with which humans share biological and cognitive ancestry, but not one other species can speak: Which of them can feel — and what do they feel? Their human spokespersons - the comparative psychologists, ethologists, evolutionists, and cognitive neurobiologists who are the world's leading experts in "mindreading" other species - will provide a sweeping panorama of what it feels like to be an elephant, ape, whale, cow, pig, dog, chicken, bat, fish, lizard, lobster, snail: This growing body of facts about nonhuman sentience has profound implications not only for our understanding of human cognition, but for our treatment of other sentient species.

|Gregory Berns: Decoding the Dog's Mind with Awake Neuroimaging

Gordon Burghardt: Probing the Umwelt of Reptiles

Jon Sakata: Audience Effects on Communication Signals

PANEL 1: Reptiles, Birds and Mammals

WORKSHOP 1: Kristin Andrews: The "Other" Problems: Mind,

Behavior, and Agency

Sarah Brosnan: How Do Primates Feel About Their Social Partners?

Alexander Ophir: The Cognitive Ecology of Monogamy

Michael Hendricks: Integrating Action and Perception in a Small

Nervous System

PANEL 2: Primates, Voles and Worms

WORKSHOP 2: Jonathan Birch: Animal Sentience and the

Precautionary Principle

Malcolm Maclver: How Sentience Changed After Fish Invaded Land

385 Million Years Ago

Sarah Woolley: Neural Mechanisms of Preference in Female

Songbird

Simon Reader: Animal Social Learning: Implications for

Understanding Others

PANEL 3: Sea to Land to Air

WORKSHOP 3: Steven M. Wise: Nonhuman Personhood

Tomoko Ohyama: Action Selection in a Small Brain (Drosophila

Maggot)

Mike Ryan: "Crazy Love": Nonlinearity and Irrationality in Mate Choice

Louis Lefebvre: Animal Innovation: From Ecology to

Neurotransmitters

PANEL 4: Maggots, Frogs and Birds: Flexibility Evolving

SPECIAL EVENT: Mario Cyr: Polar Bears

Colin Chapman: Why Do We Want to Think People Are Different?

Vladimir Pradosudov: Chickadee Spatial Cognition

Jonathan Balcombe: The Sentient World of Fishes

PANEL 5: Similarities and Differences

WORKSHOP 5 (part 1): Gary Comstock: A Cow's Concept of Her

Future

WORKSHOP 5 (part 2): Jean-Jacques Kona-Boun: Physical and

Mental Risks to Cattle and Horses in Rodeos

\author{
Joshua Plotnik: Thoughtful Trunks: Application of Elephant Cognition \\ for Elephant Conservation \\ Lori Marino: Who Are Dolphins? \\ Larry Young: The Neurobiology of Social Bonding, Empathy and \\ Social Loss in Monogamous Voles \\ Panel 6: Mammals All, Great and Small \\ WORKSHOP 6: Lori Marino: The Inconvenient Truth About Thinking \\ Chickens \\ Andrew Adamatzky: Slime Mould: Cognition Through Computation \\ Frantisek Baluska \& Stefano Mancuso: What a Plant Knows and \\ Perceives \\ Arthur Reber: A Novel Theory of the Origin of Mind: Conversations \\ With a Caterpillar and a Bacterium \\ PANEL 7: Microbes, Molds and Plants \\ WORKSHOP 7: Suzanne Held \& Michael Mendl: Pig Cognition and \\ Why It Matters \\ James Simmons: What Is It Like To Be A Bat? \\ Debbie Kelly: Spatial Cognition in Food-Storing \\ Steve Phelps: Social Cognition Across Species \\ PANEL 8: Social Space \\ WORKSHOP 8: To be announced \\ Lars Chittka: The Mind of the Bee \\ Reuven Dukas: Insect Emotions: Mechanisms and Evolutionary \\ Biology \\ Adam Shriver: Do Human Lesion Studies Tell Us the Cortex is \\ Required for Pain Experiences? \\ PANEL 9: The Invertebrate Mind \\ WORKSHOP 9: Delcianna Winders: Nonhuman Animals in Sport \\ and Entertainment \\ Carel ten Cate: Avian Capacity for Categorization and Abstraction \\ Jennifer Mather: Do Squid Have a Sense of Self? \\ Steve Chang: Neurobiology of Monkeys Thinking About Other \\ Monkeys \\ PANEL 10: Others in Mind \\ WORKSHOP 10: The Legal Status of Sentient Nonhuman Species
}

